\title{
Familial autoimmunity in pediatric patients with type 1 diabetes (T1D) and its associations with the severity of clinical presentation at diabetes diagnosis and with coexisting autoimmunity
}

\author{
Lydia Kossiva $^{1}$ (1) $\cdot$ Anastasia Korona ${ }^{1} \cdot$ Nikolitsa Kafassi $^{2} \cdot$ Spyridon Karanasios $^{1} \cdot$ Kyriaki Karavanaki $^{1}$
}

Received: 19 April 2021 / Accepted: 22 February 2022 / Published online: 7 March 2022

(c) Hellenic Endocrine Society 2022

\begin{abstract}
Purpose The aim was to evaluate the impact of familial autoimmunity on the age and severity of type 1 diabetes (T1D) presentation and on the coexistence of other autoimmune diseases.

Methods We retrospectively evaluated the medical records of 121 children/adolescents (male: 63 ) followed in our Diabetic Clinic from 2002 to 2016.

Results Seventy-six patients (62.8\%) had at least one relative with an autoimmune disease, Hashimoto's thyroiditis (49.5\%) and T1D (22.3\%) being the commonest. Children with familial autoimmunity were younger at T1D diagnosis (mean age \pm SD) $(6.766 \pm 3.75)$. Median fasting c-peptide levels at presentation were not related to familial autoimmunity. Patients with familial autoimmunity more often exhibited GADA autoantibody positivity at diagnosis. The larger the number of the patient's relatives diagnosed with an autoimmune disease, the higher were the patient's GADA levels (Spearman's rho test $=0.19$, $\mathrm{p}=0.049$ ). Children with a first-degree relative with autoimmunity had a coexisting autoimmune disorder at a significantly higher percentage $(p=0.016)$. Family history of autoimmunity was negatively associated with the presence of diabetic ketoacidosis (DKA) $(\mathrm{p}=0.024)$. Patients with a relative with T1D less frequently exhibited DKA at diagnosis $(12.8 \mathrm{vs.}$ $87.2 \%, p=0.003)$. The presence of DKA was associated with younger age $(\mathrm{p}=0.05)$ and lower $\mathrm{c}$-peptide levels $(\mathrm{p}=0.033)$. Conclusions Familial autoimmunity was present in $62.8 \%$ of children with T1D, autoimmune thyroiditis and T1D being the two most frequent familial autoimmune diseases. Familial autoimmunity reduced the risk of DKA at diagnosis, but these patients were younger and had higher levels of pancreatic autoantibodies and a greater risk of developing additional autoimmune diseases.
\end{abstract}

Keywords Diabetes mellitus $\cdot$ Childhood $\cdot$ Adolescence $\cdot$ Autoimmune disease $\cdot$ Family

\section{Introduction}

The incidence of diabetes mellitus type 1 (T1D) in childhood is estimated at $6.5 \times 10^{4}$ cases/year. Diabetic ketoacidosis (DKA) as the first presentation of the disease occurs often and is well recognized as the main cause of death at diabetes diagnosis due to severe adverse events such as electrolytic

Lydia Kossiva

lydiakossiva@hotmail.com; lydiak@med.uoa.gr

1 Diabetic Clinic, Department of Pediatrics, National and Kapodistrian University of Athens, A. Kyriakou' Children's Hospital, 'P. \&, 2nd Athens, Greece

2 Department of Immunology, 'Laiko' General Hospital, Athens, Greece disturbances, brain edema, and coma [1]. Additionally, DKA is accompanied by lower c-peptide levels due to decreased residual beta cell function [2]. During the course of diabetes, DKA is correlated with poor glycemic control and neurocognitive deficits, as well as elevated health care costs [3-6]. According to previous reports, severe DKA as the first presentation of T1D is more often observed in children without family history of T1D or other autoimmune diseases $[5,7,8]$. On the contrary, children belonging to a family with at least one first-degree relative with T1D exhibit six times lower risk of DKA as the first manifestation of T1D [9]. This is attributed to timely identification of the initial symptoms of T1D by the experienced family members. A report published by Hekkala et al. concluded that children with a family history of T1D or T2D are less likely to exhibit DKA as the first manifestation of the disease [10]. Additionally, a 
review article by Usher-Smith et al. underlines the fact that within a family with at least two members with T1D, the first to be diagnosed with the disease was at higher risk of developing severe DKA at the time of diagnosis [9]. The aim of the present study was to investigate whether DKA presence and severity at T1D diagnosis may be related to preexisting familial autoimmunity as well as to assess whether the history of other autoimmune diseases within the family constitutes a risk factor for developing T1D and/or additional autoimmune diseases during the course of diabetes. Additionally, we explored a possible link between familial autoimmunity and age at T1D diagnosis, along with the presence and titers of pancreatic autoantibodies.

\section{Methods}

The study population consisted of 121 children, adolescents, and young adults with T1D (male/female ratio: 63/58) followed up by the Diabetes and Metabolism Clinic of the Second Department of Pediatrics of the National and Kapodistrian University of Athens, 'P. \& A. Kyriakou' Children's Hospital, Athens, Greece, from January 2002 to December 2016. The participants' age ranged from 1 to $20 \mathrm{y}$, age at T1D onset was $1-15 \mathrm{y}$, and disease duration ranged from 2 months to 14.5 years. T1D and DKA diagnosis and severity were based on the International Society of Pediatric and Adolescent Diabetes (ISPAD) criteria [11]. Patients with incomplete medical records were excluded from the study. Approval of the Ethics Committee was obtained.

This is a retrospective population study. Age at T1D diagnosis, presence of pancreatic autoimmunity, and familial autoimmunity were considered as risk factors, while severity of initial T1D clinical presentation (DKA, DKA severity, or coma) was considered as the outcome. Familial autoimmunity was defined as the presence of at least one autoimmune disease either among first-degree relatives (nuclear family) or among second- and third-degree relatives (extended family).

The following parameters were extracted and documented from the patients' medical records: (a) demographic and anthropometric data; (b) DKA and its severity at T1D diagnosis; (c) disease duration; (d) fasting c-peptide levels and anti-pancreatic glutamic acid decarbozylase antibodies (GADA) and islet phosphatase 2 (IA2) antibodies at diagnosis; (e) presence of organ-specific antibodies and specifically antithyroid antibodies (anti-TPO and antiTG), celiac antibodies (anti-tTG, anti-AGA, anti-EMA$\mathrm{IgA}$, and $\mathrm{IgG}$ ) and antiparietal cell antibodies (APCA) at T1D diagnosis and during follow-up); (f) the presence of autoimmune diseases among nuclear and extended family members, according to parents' reports. A Soehnle Professional Scale was used for evaluation of anthropometric parameters, and body weight was measured at diagnosis and during each outpatient visit to the Diabetic Clinic. Body mass index (BMI) was calculated as weight (kg)/ height $(\mathrm{m})^{2}$. Evaluation of GADA and IA2 anti-pancreatic antibodies was performed using a radioimmunoassay (RIA) method, with normal upper limit $0.9 \mathrm{U} / \mathrm{ml}$ and 0.75 $\mathrm{U} / \mathrm{ml}$, respectively. Normal fasting c-peptide levels were defined as $1.77-4.68 \mathrm{ng} / \mathrm{ml}$. Antithyroid antibodies were measured using an immunoluminometric assay (ILMA). Celiac antibodies (anti-tTG, anti-AGA and anti-EMAIgA, and IgG) were measured using ELISA. Celiac disease diagnosis was based on typical small intestinal biopsy findings in patients with persistently high levels of celiacspecific antibodies. Moreover, total IgA levels were measured in all children. APCA were measured using indirect immunofluorescent assay (IFA).

\section{Statistical analysis}

Continuous variables were presented as mean value \pm SD if normally distributed, and as median value (interquartile range, IQR) if not normally distributed. Normality of continuous distributions was assessed using the Kolmogorov-Smirnov test. The categorical variables were presented as absolute (N) and relative frequency (\%). Comparison of the data was made based on tests depending on the type of variables. The chi-square test was used for comparison of categorical variables, while Fisher's exact test was used for comparison of small groups (consisting of $\leq 5$ patients). For comparison between quantitative and qualitative variables with two categories, Student's t test was used for variables following the normal distribution, and the non-parametric Mann-Whitney test for variables not following the normal distribution. One-way ANOVA and the Kruskal-Wallis test were used to compare quantitative variables with qualitative variables in more than two groups. To test the linear correlation between quantitative variables, the Spearman RHO statistical procedure was chosen. Finally, to assess the overall effect of the factors under examination related to the probability of DKA, univariate logistic regression analysis was initially carried out to export odds ratios, followed by multivariate logistic regression analysis in order to evaluate the adjusted odds ratio. The same procedure was carried out in order to assess the effect of coexistent and familial autoimmunity on the age of T1D onset. Gender, c-peptide levels, and coexistent autoimmunity were regarded as potential confounders and adjusted by multivariate modeling. Data management and all statistical analyses were performed using IBM Statistical Package for Social Sciences (SPSS) version 21 . A two-tailed $\mathrm{P}<0.05$ was considered statistically significant. 


\section{Results}

The mean \pm SD age of the patients was $11.99 \pm 4.63$ years, their median diabetes duration was 3.5 [1-6] years, their mean \pm SD age at diabetes diagnosis was $7.62 \pm 3.67$ years, while their mean $\mathrm{HbA} 1 \mathrm{c}$ during the past 12 months was $7.66 \pm 1.16 \%$. Median c-peptide levels were $0.59 \mathrm{ng} /$ $\mathrm{ml}$ (range: $0.45-0.86)$. Seventy-three percent $(73 \%)$ of patients had GADA and $71.4 \%$ had IA2 anti-pancreatic antibody positivity, while in $53 \%$ both anti-pancreatic antibodies were positive. Seventy-six patients $(62.8 \%)$ of the study group had at least one relative with an autoimmune disease within the nuclear $(52 / 121)(43 \%)$ or extended family (52/121) (43\%). Specifically, 30/121 (24.8\%) of the study group had one relative, 28/121 (23.1\%) had two relatives, 14/121 (11.6\%) had three, and 4/121 (3.3\%) had four or more relatives with autoimmune disease. Within the nuclear family, mothers were more often likely to have autoimmunity (42/121) (34.7\%) than were fathers (14/121) $(11.5 \%)$. In total, $23.1 \%$ of the patients had a parent and 9.8\% had a sibling with an autoimmune disease.

The most frequent autoimmune diseases within the nuclear or extended family were Hashimoto's thyroiditis and T1D, with $49.5 \%$ and $22.3 \%$ of the patients reporting at least one or more relatives with the disease, respectively. Other autoimmune diseases within the study group were rheumatic arthritis (4.1\%), vitiligo (4.1\%), psoriasis (3.3\%), inflammatory bowel disease (3.3\%), and multiple sclerosis (2.5\%), while other diseases such as celiac disease, Graves' disease, Sjogren syndrome, and autoimmune hepatitis were reported by $<1 \%$ of the study group.

Children with familial history of autoimmune disease within the extended family were significantly younger at T1D diagnosis compared to those without such history (mean age \pm SD: $6.76 \pm 3.75$ vs $8.26 \pm 3.51$ years, $\mathrm{p}=0.025)$. The median values of fasting c-peptide levels at first manifestation of the disease were not related to the presence/absence of familial autoimmunity [median (range): $0.58 \mathrm{ng} / \mathrm{ml}(0.43-0.83)$ vs $0.60 \mathrm{ng} / \mathrm{ml}(0.49-0.99)]$ $(\mathrm{p}=0.603)$ (Mann-Whitney U test). Patients with a history of familial autoimmunity more often significantly exhibited positivity of GADA and marginally of IA2 autoantibodies at first T1D diagnosis compared to the rest of the study group. More specifically, patients with a positive history of autoimmunity among first- and second-degree relatives exhibited significantly higher levels of GADA compared to patients without an analogous family history (median 7.56 vs. $1.58 \mathrm{U} / \mathrm{ml}, \mathrm{p}=0.041$, Mann-Whitney test) (Table 1). Additionally, the presence of autoimmunity within the nuclear family was linked to higher GADA levels (median value: $7.56 \mathrm{U} / \mathrm{ml}$ ) compared to patients without a family history (median value: $2.30 \mathrm{U} / \mathrm{ml}$ ), this being statistically significant $(p=0.042$, Mann-Whitney test). Moreover, the larger the number of relatives diagnosed with an autoimmune disease, the higher were the patient's GADA levels $(\mathrm{p}=0.049)$ (Spearman's rho test $=0.19$, $\mathrm{p}=0.049$ ).

Children with a first-degree relative with autoimmune disease had a significantly higher percentage of a coexisting autoimmune disorder compared to children without an analogous family history ( 60 vs. $36 \%, p=0.016$, Pearson chi-square). Moreover, the number of relatives with an autoimmune disease in the nuclear and extended family in total (Spearman's rho $=0.20, \mathrm{p}=0.021$ ) or, specifically, in the nuclear family (Spearman's rho $=0.24, \mathrm{p}=0.007$ ) was positively associated with the number of additional autoimmune diseases in children with T1D with statistical significance. Furthermore, multivariate regression analysis showed that patients with a history of autoimmunity within the nuclear family had a 2.7 -fold greater chance of developing another autoimmune disease during the course of the disease, independently of their age and the duration of T1D (OR: 2.7, 95\% CI: 1.18-5.96, $\mathrm{p}=0.017$ ). The larger the number of relatives with autoimmunity, the larger was the number of coexisting autoimmune diseases of a specific patient $(\mathrm{p}=0.007$, Spearman's rho 0.24).

The number of relatives with an autoimmune disease within the extended family was negatively associated with
Table 1 Titer of autoantibodies GADA and IA2 of children with T1D at diagnosis according to the presence or absence of autoimmunity within the nuclear or/and the extended family

\begin{tabular}{llllll}
\hline $\begin{array}{l}\text { Familial } \\
\text { autoimmunity }\end{array}$ & & GADA (U/ml) median (IQR) & P & $\begin{array}{l}\text { IA2 (U/ml) } \\
\text { Median (IQR) }\end{array}$ & P \\
\hline Total & Yes & $7.56(1.18-27.00)$ & $\mathbf{0 . 0 4 1}^{\text {a }}$ & $5.80(0.90-13.87)$ & $0.067^{\text {a }}$ \\
& No & $1.58(0.30-10.26)$ & & $2.82(0.11-11.03)$ & \\
Nuclear family & Yes & $7.56(2.10-21.35)$ & $\mathbf{0 . 0 4 2}^{\text {a }}$ & $4.00(0.70-10.36)$ & $0.980^{\text {a }}$ \\
& No & $2.30(0.34-21.59)$ & & $4.30(0.62-12.99)$ & \\
Extended & Yes & $5.99(0.80-29.00)$ & $0.774^{\text {a }}$ & $7.50(1.05-15.00)$ & $0.059^{\text {a }}$ \\
family & No & $4.27(0.69-20.79)$ & & $3.07(0.42-11.03)$ & \\
\hline
\end{tabular}

${ }^{a}$ Mann-Whitney test. IQR: interquartile range, GADA: glutamic acid decarboxylase autoantibodies, IA2: protein tyrosine phosphatase-like protein IA2 
the presence of DKA at diagnosis $(p=0.024$, Mann-Whitney test) compared to those without such history.

Patients with a first- or second-degree relative with T1D exhibited DKA as the first manifestation of T1D to a significantly lower percentage compared to the rest of the study group (12.8 vs. $87.2 \%, \mathrm{p}=0.003$, Pearson chi-square test). Conversely, patients with moderate/severe DKA mostly belonged to the subgroup without T1D affected relatives $(\mathrm{p}=0.004)$ (Table 2).

Univariate regression analysis showed that the presence of a positive family history of autoimmunity, as well as the number of relatives within the extended family and the number of family members with T1D had a negative correlation (protective role) with the development of DKA as the first manifestation of the disease. Additionally, the presence of DKA at diagnosis of diabetes was noted in younger patients $(p=0.05)$ and those with lower c-peptide levels $(p=0.033)$ (Table 3).

Multivariate regression analysis showed that the factors independently associated with the development of DKA at diagnosis of diabetes were patient's age $(p=0.022)$ and the number of relatives with an autoimmune disease in the
Table 2 Correlation of the presence of T1D within the family (nuclear and extended) of children with T1D and the manifestation and severity of DKA at diagnosis
Table 3 Univariate logistic regression analysis of the factors linked to the manifestation of DKA at T1D diagnosis

\begin{tabular}{|c|c|c|c|c|c|c|}
\hline \multirow[t]{2}{*}{ DKA } & \multicolumn{2}{|c|}{ T1D (extended family) } & \multirow[b]{2}{*}{$\mathbf{P}$} & \multicolumn{3}{|c|}{ T1D (nuclear family) } \\
\hline & $\begin{array}{l}\text { Yes } \\
\mathbf{N}(\%)\end{array}$ & $\begin{array}{l}\text { No } \\
\text { N(\%) }\end{array}$ & & $\begin{array}{l}\text { Yes } \\
\mathbf{N}(\%)\end{array}$ & $\begin{array}{l}\text { No } \\
\mathbf{N}(\%)\end{array}$ & $\mathbf{P}$ \\
\hline No & $14(36.8)$ & $24(63.2)$ & $\mathbf{0 . 0 0 3}^{\mathrm{a}}$ & $5(13.2)$ & $33(86.8)$ & $0.112^{\mathrm{a}}$ \\
\hline Yes & $10(12.8)$ & $68(87.2)$ & & $3(3.8)$ & $75(96.2)$ & \\
\hline Mild & $4(21.1)$ & $15(78.9)$ & $0.014^{\mathrm{a}}$ & $2(10.5)$ & $17(89.5)$ & $0.107^{\mathrm{a}}$ \\
\hline Moderate & $4(14.8)$ & $23(85.2)$ & & $1(3.7)$ & $26(96.3)$ & \\
\hline Severe & $2(6.3)$ & $30(93.8)$ & & $0(0)$ & $\begin{array}{l}32 \\
(100)\end{array}$ & \\
\hline Absent/mild & $18(31.6)$ & $39(68.4)$ & $0.004^{\mathrm{a}}$ & $7(12.3)$ & $50(87.7)$ & $0.031^{b}$ \\
\hline Moderate/severe & $6(10.2)$ & $53(89.8)$ & & $1(1.7)$ & $58(98.3)$ & \\
\hline
\end{tabular}

${ }^{a}$ Pearson chi-square. ${ }^{b}$ Fisher's exact test

\begin{tabular}{lccc}
\hline & OR* & $\mathbf{9 5 \%}$ CI** & P \\
\hline Gender (male/female) & 1.30 & $0.59-2.83$ & 0.509 \\
Age at T1D diagnosis (y) & 0.92 & $0.82-1.02$ & 0.143 \\
Age category of T1D diagnosis (y) & & & \\
$12.1-15$ & & & \\
$1-2$ & 10.80 & $1.00-116.99$ & $\mathbf{0 . 0 5 0}$ \\
$2,1-12$ & 2.47 & $0.70-8.75$ & 0.159 \\
c-peptide & 0.26 & $0.07-0.89$ & $\mathbf{0 . 0 3 3}$ \\
GADA & 1.00 & $0.99-1.00$ & 0.829 \\
IA2 & 0.99 & $0.99-1.00$ & 0.386 \\
Coexisting autoimmunity at diagnosis (yes/no) & 0.69 & $0.22-2.12$ & 0.523 \\
Coexisting autoimmunity & 1.03 & $0.42-2.48$ & 0.945 \\
(yes/no) & & & \\
Number of coexisting autoimmune diseases & 1.33 & $0.66-2.67$ & 0.421 \\
Familial autoimmunity(nuclear and extended) (yes/no) & 0.74 & $0.33-1.66$ & 0.480 \\
Number of relatives (nuclear and extended) & 0.80 & $0.58-1.10$ & 0.181 \\
Familial autoimmunity (nuclear) (yes/no) & 0.95 & $0.43-2.10$ & 0.912 \\
Number of relatives (nuclear family) & 1.10 & $0.64-1.90$ & 0.721 \\
Familial autoimmunity (extended family) (yes/no) & 0.45 & $0.20-0.99$ & $\mathbf{0 . 0 4 9}$ \\
Number of relatives (extended family) & 0.61 & $0.39-0.96$ & $\mathbf{0 . 0 3 5}$ \\
Family history of T1D (nuclear and extended) (yes/no) & 0.25 & $0.09-0.64$ & $\mathbf{0 . 0 0 4}$ \\
Number of relatives with T1D (nuclear and extended) & 0.27 & $0.12-0.63$ & $\mathbf{0 . 0 0 2}$ \\
Family history of T1D (nuclear family) (yes/no) & 0.26 & $0.06-1.17$ & 0.080 \\
\hline
\end{tabular}

*OR: odds ratio, **CI:95\% confidence interval, GADA: glutamic acid decarboxylase autoantibodies. IA2: protein tyrosine phosphatase-like protein IA2 
extended family $(\mathrm{p}=0.010)$. In particular, children aged 1-2 years old were 20 -fold more likely on being diagnosed with diabetes to develop DKA in comparison with those aged 12,1-15 years old, independently of other factors (OR: 21.42, 95\% CI: 1.55-295.63, P: 0.022). Moreover, for every additional relative with autoimmunity in the extended family, the likelihood of developing DKA was reduced by $48 \%$, independently of the presence of other factors (OR: 0.52 , 95\% CI: 0.32-0.85, P: 0.010) (Table 4).

\section{Discussion}

In the present study, we retrospectively evaluated the prevalence of familial autoimmunity in the relatives of children and adolescents with T1D and its effect on the development and severity of DKA at diagnosis of diabetes. We also evaluated the correlation of familial autoimmunity with laboratory parameters (c-peptide and pancreatic autoantibodies), as well as its association with the development of additional autoimmune diseases in the patients. There are very few studies to date assessing the frequencies of familial autoimmune diseases in the relatives of children and adolescents with T1D, and, to our knowledge, only one of them has reported on its association with the presence and severity of DKA at diagnosis of diabetes or pancreatic autoimmunity [12].

Several common genetic loci along with environmental factors can predispose to the manifestation of either a specific type or a spectrum of autoimmune diseases within the members of the family [13]. T1D is an excellent example of an autoimmune disease that is linked to other autoimmune diseases, either in the proband or in the family members. The majority of our study group $(62.8 \%)$ reported at least one relative with an autoimmune disease, while $43 \%$ had at least one first-degree relative and another $43 \%$ had a

Table 4 Multivariate regression analysis of the factors related to DKA at T1D diagnosis

\begin{tabular}{lcll}
\hline & Adjusted OR* & 95\% CI** & P \\
\hline Age at T1D diagnosis (y) & & & \\
$1-2$ & 21.42 & $1.55-295.63$ & $\mathbf{0 . 0 2 2}$ \\
$2.1-12$ & 2.65 & $0.70-9.97$ & 0.149 \\
Gender & 1.39 & $0.59-3.24$ & 0.442 \\
$\begin{array}{l}\text { Number of relatives with } \\
\quad \text { autoimmunity (extended } \\
\quad \text { family) }\end{array}$ & 0.52 & $0.32-0.85$ & $\mathbf{0 . 0 1 0}$ \\
$\begin{array}{l}\text { Coexisting autoimmunity } \\
\quad(y e s / n o)\end{array}$ & 1.20 & & \\
$\begin{array}{l}\text { Familial autoimmunity } \\
\quad(\text { nuclear family) (yes/no) }\end{array}$ & 1.11 & $0.45-3.16$ & 0.713 \\
\hline
\end{tabular}

*OR: adjusted odds ratio, **95\% CI: confidence interval second- or third-degree relative with autoimmunity. Alhonen et al. reported that $22 \%$ of children with T1D had a positive autoimmunity history within the nuclear family compared to healthy controls (12.9\%). The aforementioned percentage was much higher among both nuclear and extended family members compared to controls ( 72 vs. $62.2 \%$, respectively). [14] Another two studies by Parkkola et al. and Anaya et al. reported in children with T1D a frequency of at least one first-degree relative with an autoimmune disease of $11.2 \%$ and $25.5 \%$, respectively, compared to $9 \%$ of controls [15, 16]. The above frequencies were lower than those reported in our study (43\%), this possibly attributable to methodological differences among studies. [14, 16].

The most common autoimmune diseases within the nuclear and extended families were Hashimoto's thyroiditis (49.5\%) and T1D (22.3\%), followed by rheumatic arthritis $(4.1 \%)$, vitiligo $(4.1 \%)$, psoriasis $(3.3 \%)$, inflammatory bowel disease $(3.3 \%)$, and multiple sclerosis $(2.5 \%)$, while other diseases such as celiac disease, Graves' disease, Sjogren syndrome, and autoimmune hepatitis were reported among $<1 \%$ of the study group. Only a few reports have evaluated the frequency of familial autoimmunity in T1D patients, especially within the extended family [16-18].

A large study from Sweden reported that among 21,168 children with T1D, the most prevalent familial autoimmune diseases were celiac disease, autoimmune thyroiditis, Graves' disease, Addison's disease, and rheumatic disease [17]. In line with our results are those of Parkkola et al. who noted that the commonest familial autoimmune diseases among first/second-degree relatives were autoimmune thyroiditis $(17 \%)$, rheumatic disease (12\%), and celiac disease (5.2\%). In the latter study, children with familial history of T1D were not included [16]. Similar results have been reported in two other studies $[14,15]$. Majeed et al. found that $27.1 \%$ of children with T1D had a relative with T1D and $15.6 \%$ a relative with thyroiditis. [18] Thus, specific autoimmune diseases run in families with T1D affected patients, which indicates the possible effect of common pathogenetic loci on the development of these diseases.

The presence of familial autoimmunity in our study was not associated with the patients' fasting c-peptide levels at T1D diagnosis. This could be explained by the possible dual role played by familial autoimmunity in T1D manifestation. Specifically, the increased autoimmunity load in the family could result in a more aggressive form of disease presentation, expressed by low c-peptide levels. On the other hand, within the families with autoimmune diseases, especially T1D, there is a high degree of awareness regarding suspected diabetes symptoms, leading to a timely diagnosis at an earlier stage of the disease at which there are adequate remaining b-cells and thus relatively higher levels of c-peptide. Our findings are in accordance with previous reports in the international literature. [12, 19] In particular, the study 
by Karges B et al., including 3765 patients with familial type T1D and 53,606 patients with sporadic T1D identified by the Diabetes Prospective Follow-up Registry, reported that patients with familial type T1D were diagnosed at a younger age, while they had lower HbA1c levels, lower prevalence of DKA at diagnosis, and higher prevalence of associated autoimmune diseases compared to those with sporadic T1D [12].

In the present study, GADA titers were higher in patients with autoimmunity within both the nuclear and the extended family, while the larger the number of the patient's relatives diagnosed with autoimmunity, the higher were the patient's levels of GADA. According to previous reports, positive GADA autoantibodies have been associated with the patient presenting coexisting autoimmunity, particularly thyroid and gastric autoimmunity (thyrogastric autoimmunity), and have been considered as a marker of the future development of thyrogastric autoimmunity in T1D patients $[16,20]$. Our data confirm the results from the study by DeBlock et al. showing that the presence of thyrogastric antibodies in firstdegree relatives of patients with T1D is associated with the patient's autoantibody status [21]. The association of GADA with the presence of thyrogastric antibodies in patients with T1D may be explained by the hypothesis that the pancreas, thyroid gland, and stomach share common antigens [22, 23]. Once a $\mathrm{T}$ cell response to GADA has been primed in the pancreas, the resulting activated $\mathrm{T}$ cells initiate damage of other neuroendocrine tissues containing the same or similar enzymes [23].

Moreover, Brorsson et al. reported that specific common genetic loci are responsible for the pathogenesis of T1D and the production of organoid autoantibodies such as GADA, IA2, anti-TPO, and anti-TG, and these loci predispose to several autoimmune diseases within families [24]. In contrast, Parkkola et al. did not find any correlation between the presence of anti-pancreatic autoantibodies and familial autoimmunity. The latter discrepancies with the present study could be due to methodological differences [16].

Regarding the presence of family history of T1D, according to our results, $22.3 \%$ of the study group had at least one relative with T1D within the nuclear and/or the extended family (7.4\% nuclear and 19\% extended). Similarly, a previous paper by Parkkola et al. reported that $10-12 \%$ of patients with T1D had at least one first-degree and $5-16 \%$ one second-degree relative with T1D [15]. There is only one study, from Finland, reporting that a much higher percentage (50\%) of children with T1D had one or more relatives within the extended family. This could be explained by the high prevalence of the disease in the latter country [14].

Another significant finding of our study is that patients with positive family history of autoimmunity, and especially of T1D, were younger at the time of diagnosis compared to the rest of the study group. The higher the number of relatives diagnosed with an autoimmune disease within the extended family, the younger was the age of T1D manifestation. These findings are in agreement with the study by Milluzzo et al., who reported that T1D patients with a family history of T1D represent a distinct manifestation of autoimmune diabetes in that they are diagnosed at a younger age and have an increased risk of development of additional autoimmune diseases in comparison to those with sporadic T1D [25].

The latter observation is in accordance with the existing literature, indicating that genetic factors may play a significant role in the pathogenesis of T1D, resulting in a more aggressive autoimmune process [12, 21, 25-27]. On the other hand, multifactorial logistic regression analysis of our data showed that the presence of T1D family history acted protectively against DKA manifestation due to timely diagnosis. Regarding the effect of the recent COVID19 pandemic on T1D diagnosis, all studies agree that the frequency and severity of DKA among newly diagnosed children and adolescents with T1D has increased, although there are controversial reports on pediatric T1D incidence, with some studies reporting a reduction, others an increase, and others no change [28-30]. The presumed increase in the frequency and severity of DKA at T1D diagnosis has been attributed by the authors either to delayed attendance, and therefore late diagnosis, at the outpatient clinic due to the fear of SARS-CoV-2 exposure or to the diabetogenic effect of the virus itself $[28,29]$. These observations highlight the importance of early diagnosis of T1D for the prevention of DKA development.

In the present study, we also found a correlation between familial and coexisting autoimmunity. Children with a firstdegree relative with autoimmune disease had a three-fold higher risk of experiencing a second autoimmune disease during the course of T1D, independently of the patient's age or the duration of the disease (OR:2.72, $\mathrm{p}=0.021)$. Additionally, children with coexisting autoimmunity had a higher proportion (i.e., $>3$ ) of relatives with autoimmunity compared to the rest of the study group $(p=0.026)$, this being in accordance with previous reports [12, 25, 31]. A thorough follow-up that includes monitoring for possible coexisting autoimmune diseases is recommended in T1D patients, especially those with a family history of autoimmunity and prompt advice should be offered to the relatives of these patients concerning the higher risk for development of an autoimmune disease in the future. In fact, the TEDDY (The Environmental Determinants of Diabetes in the Young) study (2014), which examined the prevalence of pancreatic autoimmunity among the first-degree relatives of T1D children, showed that the identification of three anti-pancreatic antibodies among the siblings of T1D children (i.e., children with high genetic risk for T1D) led to a significant degree to early diabetes diagnosis and prevention of DKA (TEDDY 2011) [32, 33]. The importance of DKA prevention at T1D 
diagnosis has been analyzed by Kakleas et al. who have demonstrated that severe DKA might be causally associated with future development of polyautoimmunity in these patients [34]. The authors also suggest that the development of polyautoimmunity in children with T1D might be due to the more aggressive autoimmune endotype of the disease [35]. To conclude, while information on the presence of familial autoimmunity is of extreme importance for early T1D diagnosis and possible DKA prevention, it could also be used as a marker for the future development of coexisting autoimmunity.

The limitations of our study are its retrospective character and the relatively small number of study participants. Nevertheless, our data should be considered representative as they come from a referral center for the regions of Central and Southern Greece and the islands. Another limitation of the present study is that only two anti-pancreatic antibodies were initially assessed (GADA and IA2) in the total study population, and in the case of negative results, we proceeded to the investigation of the remaining anti-pancreatic antibodies (ICA, IAA, and ZnT8), as previously suggested [36]. GADA and IA2 anti-pancreatic antibodies are, however, the most frequently occurring ones and their combination has been shown to be the best predictor for future T1D development [37].

A strength of the present study is that is among the very few in the literature to assess a large number of [20] autoimmune diseases in the nuclear and extended family and it is the only one to assess the association of familial autoimmunity with the development and severity of DKA at diabetes presentation.

\section{Conclusions}

In the present study, familial autoimmunity was present in more than half (62.8\%) of children with T1D, with autoimmune thyroiditis being the most frequent familial autoimmune disease, followed by T1D. However, T1D patients with familial autoimmunity were younger at diagnosis and had a higher prevalence, higher titers of pancreatic autoantibodies, and a greater risk of developing another autoimmune disease during the course of the disease. The clinical importance of our findings is significant, since identifying the factors related to DKA development at T1D diagnosis may lead to the reduction of its frequency and severity. Our study has also shown that the presence of familial autoimmunity might act as a protective factor in relation to DKA at T1D diagnosis and its severity.

It is therefore evident that in all children with newly diagnosed T1D, a detailed family history should be taken and advice should be offered to both the patient and the family on the risks of developing an additional autoimmune disease. Moreover, all patients should have a regular, annual laboratory evaluation to monitor for potential additional autoimmune diseases so as to avoid any delayed diagnosis. Further larger studies are necessary to shed further light on the above findings.

Author contribution All authors contributed to the study.

- Karavanaki Kyriaki contributed to conceptualization.

- Korona Anastasia provided methodology.

- Korona Anastasia and Kossiva Lydia performed formal analysis and investigation.

- Kossiva Lydia performed writing, original draft preparation.

- Karavanaki Kyriaki and Kossiva Lydia performed writing, review, and editing.

- Korona Anastasia and Karanasios Spyridon done data collection and analysis.

- Kafassi Nikolitsa carried out laboratory analysis.

All authors read and approved the final manuscript.

Data availability Patients' charts are available.

Code availability Not applicable.

\section{Declarations}

Ethical approval This retrospective chart review study involving human participants was in accordance with the ethical standards of the institutional and national research committee and with the 1964 Helsinki Declaration and its later amendments or comparable ethical standards. The Human Investigation Committee (IRB) of the "P\&A Kyriakou" Children's Hospital approved this study.

Informed consent Informed consent was obtained from the patients or their legal guardians.

Conflict of interest All authors declare no conflict of interest.

\section{References}

1. Usher-Smith JA, Thompson M, Ercole A, Walter FM (2012) Variation between countries in the frequency of diabetic ketoacidosis at first presentation of type 1 diabetes in children: a systematic review. Diabetologia 55:2878-2894

2. Cesur M and Sayin I (2013) Diabetic Ketoacidosis. In:Type 1 Diabetes, Escher A (Ed.), InTech. Available from: https://www. intechopen.com/books/type-1-diabetes/diabetic-ketoacidosis.

3. Ghetti S, Lee KJ, Sims CE, Demster DM, Glaser NS (2010) Diabetic Ketoacidosis and Memory Dysfunction in Children with Type 1 Diabetes. J Pediatr 156:109-114. https://doi.org/10.1016/j. jeds.2009.07.054

4. Jessup AB, Grimley MB, Meyer E et al (2015) Effects of Diabetic Ketoacidosis on Visual and Verbal Neurocognitive Function in Young Patients Presenting with New-Onset Type 1 Diabetes. J Clin Res Pediatr Endocrinol 7:203-210

5. Fredheim S, Johannesen J, Johansen A et al (2013) Diabetic ketoacidosis at the onset of type 1 diabetes is associated with future HbA1c levels. Diabetologia 56:995-1003. https://doi.org/ 10.1007/s00125-013-2850-z 
6. Shalitin S, Fisher S, Yackobovitz-Gavan M, de Vries L, Lazar L, Lebenthal Y, Phillip M (2017) Ketoacidosis at onset of type 1 diabetes is a predictor of long-term glycemic control. Pediatr Diabetes 19:320-328. https://doi.org/10.1111/pedi.12546

7. Klingensmith G J, Tamborlane WV, Wood J, Wong-Jacobson S, Beck RW (2013) Diabetic Ketoacidosis at Diabetes Onset: Still an All Too Common Threat in Youth. The Journal of Pediatrics 162:330 - 334.doi:https://doi.org/10.1016/j.jpeds.2012.06.058.

8. de Vries L, Oren L, Lazar L, Lebenthal Y, Shalitin S, Phillip M (2013) Factors associated with diabetic ketoacidosis at onset of Type 1 diabetes in children and adolescents. Diabet Med 30:1360-1366

9. Usher-Smith J.A., Thompson M., Sharp S., Walter FM (2011) Factors associated with the presence of diabetic ketoacidosis at diagnosis of diabetes in children and young adults: a systematic review. BMJ 343:d4092.

10. Hekkala A, Ilonen J, Knip M, Veijola R, the Finnish Paediatric Diabetes Register, (2011) Family history of diabetes and distribution of class II HLA genotypes in children with newly diagnosed type 1 diabetes: effect on diabetic ketoacidosis. Eur J Endocrinol 165:813-817

11. Mahmud FH, Elbarbary NS, Fröhlich-Reiterer E, Holl RW, Kordonouri O, Knip M et al. (2018) ISPAD Clinical Practice Consensus Guidelines: Other complications and associated conditions in children and adolescents with type 1 diabetes.Pediatr 19(Suppl 27): 275-286. doi:https://doi.org/10.1111/pedi.12740.

12. Karges B, Prinz N, Placzek K, Datz N, Papsch M, Strier U, Agena D, Bonfig W, Kentrup H, Holl RW (2021) A Comparison of Familial and Sporadic Type 1 Diabetes Among Young Patients. Diabetes Care 44:1116-1124. https://doi.org/10.2337/ dc20-1829

13. Castiblanco J., Sarmiento-Monroy JC., Mantilla RD, Rojas-Villarraga A, Anaya JM (2015) Familial Aggregation and Segregation Analysis in Families Presenting Autoimmunity, Polyautoimmunity, and Multiple Autoimmune Syndrome. Journal of Immunology Research. 2015, 10 pages. https://doi.org/10.1155.2015/572353

14. Alhonen S, Korhonen S, Tapanainen PA, Knip M, Veijola R (2011) Extended Family History of Diabetes and Autoimmune Diseases in Children With and Without Type 1 Diabetes. Diabetes Care 34:115-117

15. Anaya JM, Castiblanco J, Tobón GJ, García J, Abad V, Cuervo H, Velásquez A, Angel ID, Vega P, Arango AJ (2006) Familial clustering of autoimmune diseases in patients with type 1 diabetes mellitus. Autoimmun 26:208-214

16. Parkkola A, Harkonen T, Ryhanen SJ, Ilonen J, Knip M, Register FPD (2013) Extended family history of autoimmune diseases and phenotype of children with newly diagnosed type 1 diabetes. Eur J Endocrinol 169:171-178

17. Hemminki K, Li X, Sundquist J, Sundquist K (2009) Familial association between type 1 diabetes and other autoimmune and related diseases. Diabetologia 52:1820-1828

18. Majeed AAS, Mea HK (2011) Risk Factors for Type 1 Diabetes Mellitus among Children and Adolescents in Basrah. Oman Med J 26:189-195

19. Samuelsson U, Lindblad B, Carlsson A et al (2013) Residual beta cell function at diagnosis of type 1 diabetes in children and adolescents varies with gender and season. Diabetes Metab Res Rev 29:85-89

20. Kakleas K, Kostaki M, Critselis E, Karayianni C, Giannaki M, Anyfantakis K, Haramaras I, Fotinou A, Papathanasiou A, Karavanaki K( 2012) Gastric autoimmunity in children and adolescents with type 1 diabetes: a prospective study. Horm Res Paediatr $77: 121-126$.

21. De Block CE, LeeuwIH De, Decochez K, Winnock F, Van Autreve J, Van Campenhout CM et al (2001) The presence of thyrogastric antibodies in first degree relatives of type 1 diabetic patients is associated with age and proband antibody status. J Clin Endocrinol Metab 86:4358-4363

22. De Block CE, De Leeuw IH, Rooman RP, Winnock F, Du Caju MV, Van Gaal LF (2000) Gastric parietal cell antibodies are associated with glutamic acid decarboxylase- 65 antibodies and the HLA DQA1*0501-DQB1*0301 haplotype in Type 1 diabetes mellitus. Belgian Diabetes Registry Diabet Med 17:618-622

23. Esteban M, Baxter AG (2001) Polyspecificity of autoimmune responses in type 1 (autoimmune) diabetes. Clin Exp Immunol 126:184-186

24. Brorsson CA, Pociot F (2015) The Type 1 Diabetes Genetics Consortium (2015) Shared Genetic Basis for Type 1 Diabetes, Islet Autoantibodies, and Autoantibodies Associated With Other Immune-Mediated Diseases in Families With Type 1 Diabetes. Diabetes Care 38:S8-S13. https://doi.org/10.2337/dcs15-2003

25. Milluzzo A, Falorni A, Brozzetti A, Pezzino G, Tomaselli L, Tumminia A, Frittitta L, Vigneri R, Sciacca L (2021) Risk for Coexistent Autoimmune Diseases in Familial and Sporadic Type 1 Diabetes is Related to Age at Diabetes Onset. Endocr Pract 27:110-117

26. Bizzarri C, Paladini A, Benevento D, Fierabracci A, Cappa M (2015) Family history and ethnicity influencing clinical presentation of type 1 diabetes in childhood. J Endocrinol Invest 38:1141-1143

27. Veijola R, Reijonen H, Vähäsalo $P$, Sabbah E, Kulmala P, Ilonen J, Akerblom HK, Knip M, and the Childhood Diabetes in Finland (DiMe) Study Group (1996) HLA-DQB1-defined Genetic Susceptibility, Beta Cell Autoimmunity, and Metabolic Characteristics in Familial and Nonfamilial Insulin dependent Diabetes Mellitus. J Clin Invest 98:2489-2495

28. Rabonne I, Schiaffini R, Cherubini V, Maffeis C, Scaramuzza A (2020) Diabetes Study Group of the Italian Society for Pediatric Endocrinology and Diabetes. Has COVID-19 delayed the diagnosis and worsened the presentation of type 1 diabetes in children? Diabetes Care 43:2870-2872. https://doi.org/10.2337/dc20-1321

29. Unsworth R, Wallace S, Oliver NS, Yeung S, Kshirsagar A, Naidu H, Min Wai Kwong R, Kumar P, Logan KM (2020) New onset type 1 diabetes in children during Covid-19: Multicenter regional findings in the U.K. Diabetes Care 43:e170-e171

30. Tittel SR, Rosenbauer J, Kamrath C, Ziegler J, Reschke F, Hammersen J, Mönkemöller K, Pappa A, Kapellen T, Holl RW (2020) DPV Initiative. Did the COVID-19 lockdown affect the incidence of pediatric type 1 diabetes in Germany? Diabetes Care 43:e172e173. https://doi.org/10.2337/dc20-1633

31. Hanukoglu A, Mizrachi A, Dalal I, Admoni O, Rakover Y, Bistritzer Z, Levine A, Somekh E, Lehmann D, Tuval M, Boaz M, Golander A (2003) Extrapancreatic autoimmune manifestations in type 1 diabetes patients and their first-degree relatives: a multicenter study. Diabetes Care 26:1235-1240

32. Elding Larsson EH, Vehik K, Gesualdo P, Akolkar B, Hagopian W, Krischer J, Lernmark Å, Rewers M, Simell O, She JX, Ziegler A, Haller MJ; TEDDY Study Group (2014) Children followed in the TEDDY study are diagnosed with type 1 diabetes at an early stage of disease. Pediatr Diabetes 15:118-126

33. Elding Larsson EH, Vehik K, Bell R, Dabelea D, Dolan L, Pihoker C, Knip M, Veijola R, Lindblad B, Samuelsson U, Holl R, Haller MJ; TEDDY Study Group; SEARCH Study Group; Swediabkids Study Group; DPV Study Group; Finnish Diabetes Registry Study Group (2011) Reduced prevalence of diabetic ketoacidosis at diagnosis of type 1 diabetes in young children participating in longitudinal follow-up. Diabetes Care 34:2347-2352

34. Kakleas K, Basatemur E, Karavanaki K (2021) Association between severity of diabetic ketoacidosis at diagnosis and multiple autoimmunity in Children With type 1 diabetes mellitus: A study from a Greek tertiary centre. Can J Diabetes 45:33-38.e2. https:// doi.org/10.1016/j.jcjd.2020.05.003 
35. Battaglia M, Ahmed S, Anderson MS et al (2020) Introducing the endotype concept to address the challenge of disease heterogeneity in type 1 diabetes. Diabetes Care 43:5-12

36. Kawasaki E (2014) Type 1 diabetes and autoimmunity. Clin Pediatr Endocrinol 23:99-105

37. Ota T, Takamura T, Nagai Y, Bando Y, Usuda R (2005) Significance of IA-2 antibody in Japanese type 1 diabetes: its association with GAD antibody. Diabetes Res Clin Pract 67:63-69
Publisher's note Springer Nature remains neutral with regard to jurisdictional claims in published maps and institutional affiliations. 